\title{
Association of gene polymorphisms in ABO blood group chromosomal regions and menstrual disorders
}

\author{
YONG SU, GUI-LIAN KONG, YA-LI SU, YAN ZHOU, LI-FANG LV, QIONG WANG, BAO-PING HUANG, \\ RUI-ZHI ZHENG, QUAN-ZHONG LI, HUI-JUAN YUAN and ZHI-GANG ZHAO \\ Department of Endocrinology, People's Hospital of Zhengzhou University (Henan Province People's Hospital), \\ Zhengzhou, Henan 450003, P.R. China
}

Received May 12, 2014; Accepted March 24, 2015

DOI: $10.3892 /$ etm.2015.2416

\begin{abstract}
This study aimed to investigate whether single nucleotide polymorphisms (SNPs) located near the gene of the ABO blood group play an important role in the genetic aetiology of menstrual disorders (MDs). Polymerase chain reaction-ligase detection reaction technology was used to detect eight SNPs near the ABO gene location on the chromosomes in 250 cases of MD and 250 cases of normal menstruation. The differences in the distribution of each genotype, as well as the allele frequency in the normal and control groups, were analysed using Pearson's $\chi^{2}$ test to search for disease-associated loci. SHEsis software was used to analyse the linkage disequilibrium and haplotype frequencies and to inspect the correlation between haplotypes and the disease. Compared with the control group, the experimental group exhibited statistically significant differences in the genotype distribution frequencies of the rs657152 locus of the ABO blood group gene and the rs17250673 locus of the tumour necrosis factor cofactor 2 (TRAF2) gene, which is located downstream of the ABO gene. The allele distribution frequencies of rs657152 and rs495828 loci in the ABO blood group gene exhibited significant differences between the groups. Dominant and recessive genetic model analysis of each locus revealed that the experimental group exhibited statistically significant differences from the control group in the genotype distribution frequencies of rs657152 and rs495828 loci, respectively. These results indicate that the ABO blood group gene and TRAF2 gene may be a cause of MDs.
\end{abstract}

Correspondence to: Dr Yong Su, Department of Endocrinology, People's Hospital of Zhengzhou University (Henan Province People's Hospital), 7 Weiwu Road, Zhengzhou, Henan 450003, P.R. China E-mail: ynogsu@163.com

Key words: ABO blood group gene, gene polymorphism, menstrual disorders

\section{Introduction}

Menstruation is the periodic endometrial shedding and bleeding that accompanies ovarian cyclical ovulation. Regular menstruation is one of the signs of mature female reproductive function. Menstrual disorders (MDs) are common gynaecological diseases that not only affect the health and quality of life of females but also increase the potential risks of certain gynaecological diseases (1-4). In recent years, research has increasingly focused on the genetic factors that cause MDs. Taylor et al (5) found that polymorphisms in the progesterone receptor gene were associated with young menarche ages and excessively short menstrual cycles. The probability of premenstrual syndrome (PMS) has been found to be higher in identical twins than in fraternal twins (6), and mutation of the follicle-stimulating hormone receptor (FSH-R) gene may lead to amenorrhoea, infertility or premature ovarian failure (7). These findings indicate that genetic susceptibility is significant in the occurrence of MDs. Furthermore, a predisposing gene of polycystic ovary syndrome, which is normally accompanied by MDs (8), is located in 9q33.3 (9). The ABO gene is located on chromosome $9 \mathrm{q} 34.2$; thus, the association of the blood type system with MDs may involve the ABO gene. Previous studies have described the correlation of the ABO blood group with ovarian reserve functions and infertility. It has been reported that antigen A may be a protective factor of ovarian reserve capacity and that type $\mathrm{O}$ blood decreases the risk of ovarian reserve capacity more than blood types A and AB (10-12). It has been proposed that type $\mathrm{O}$ blood is associated with infertility (13). In certain studies, pregnant females with type A blood were discovered to be more prone to ovarian hyperstimulation than females with type O blood $(14,15)$. These studies indicate that the ABO blood system is closely associated with diseases of the female reproductive system. As MDs are common gynaecological diseases that reflect the ovarian state, the occurrence of MDs may be associated with the ABO blood group system.

To verify these hypotheses and evaluate whether single nucleotide polymorphisms (SNPs) are associated with MDs, the $\mathrm{ABO}$ blood group gene and eight related gene loci, which are located at upstream and downstream sites of the ABO gene, were examined in the present study. 


\section{Materials and methods}

Subjects. A total of 1,035 females of childbearing age were enrolled by the Reproductive Health Research Institute of Henan Provincial People's Hospital (Jinshui, China). Among the subjects, 532 cases had regular menstruation and 503 cases had MDs. All patients were not biological relations of any other patient. The females with regular menstruation were set as the control group, whereas females with MDs were set as the experimental group. Random sampling was performed to select 250 cases from each group. This study was conducted in accordance with the Declaration of Helsinki and with approval from the Ethics Committee of Henan Provincial People's Hospital. Written informed consent was obtained from all participants.

Judgment criteria. Menstrual cycles $<21$ days and $>35$ days were defined as polymenorrhoea and oligomenorrhoea, respectively. Regarding menstrual blood volume, daily usages of $<2$ and $>5$ sanitary towel pieces, each fully soaked by menstrual blood, were defined as hypomenorrhoea and hypermenorrhoea, respectively. Menstruation durations that were not between three and seven days were considered abnormal. The presence of dysmenorrhoea was defined as the occurrence before, during or after menstruation of lower abdominal pain and a feeling of fullness and bloating accompanied by backache or other symptoms that seriously affected the quality of life.

Females with normal menstrual cycles, blood volume and no dysmenorrhoea were considered normal, whereas females with any of the above symptoms were considered MD patients (16).

Samples. The questionnaire was co-designed with epidemiological, statistical and endocrinological experts. The questionnaire included general situations, menstrual characteristics and relevant family history. The questionnaire was completed by the respondents. Thereafter, a specialist individually asked the patients for verification and supplemental information.

The medical staff of Henan Provincial People's Hospital conducted ABO blood group detection by the slide method. Anti-A and -B antibodies were purchased from the Biomedical Engineering Center of Hebei Medical University (Shijiazhuang, China). A total of $2 \mathrm{ml}$ venous blood was drawn from each participant and was placed into a 2-ml disposable dipotassium ethylenediamine-N,N,N',N'-tetraacetic acid-containing human blood vial, followed by covering and mixing. The vial was placed into a freezer at $-80^{\circ} \mathrm{C}$ for future extraction of DNA.

DNA extraction and detection. A $1 \mathrm{ml}$ blood genomic DNA extraction kit based on a centrifugation-column method (Shanghai Lifefeng Biotechnology Co. Ltd., Shanghai, China) was used to extract DNA from the blood samples, and a NanoDrop-1000 UV/VIS spectrophotometer (Thermo Fisher Scientific Inc., Waltham, MA, USA) was used to ensure that the concentrations of each DNA sample were $\geq 50 \mathrm{ng} / \mu \mathrm{l}$, the purity at optical density (OD) 260/280 was 1.7-2.0 and at OD $260 / 230$ was $>1.5$. Polymerase chain reaction-ligase detection (PCR-LDR) technology was used to genotype the loci. The PCR-LDR was completed by Shanghai Generay Biotech Co. Ltd. (Shanghai, China) with an accuracy rate of up to $98 \%$.
Choice of SNP loci. The ABO gene is located on chromosome 9q34.2 (NM_020469), and the bases are located at positions 136130563 to 136150630 . Eight loci near the ABO gene were chosen. rs657152 is inside one of the ABO gene introns, and rs495828 is located upstream of the ABO gene. Downstream of the ABO gene, four loci of the tumour necrosis factor receptor cofactor 2 (TRAF2) gene, namely, rs7852970, rs4448378, rs10781522 and rs17250673, and two loci of the patatin-like phospholipase domain containing 7 (PNPLA7) gene, namely, rs61754920 and rs11137410, were selected. SNP information was downloaded from the website of the International HapMap for the Han Chinese in Beijing, China (CHB) population (17). The SNP information met the minimum allele frequency (MAF) standard of $>0.05$.

Data analysis. Counting data are expressed as percentages, and measurement data are expressed as mean \pm standard deviation. The Hardy-Weinberg genetic equilibrium law was performed to test the genotype distribution of the experimental and control groups. Pearson's $\chi^{2}$ test was used to test the differences of genotype frequencies and allele frequency distributions between the two groups. The linkage disequilibrium and haplotype frequency of each locus was analysed by SHEsis software (18) to investigate the relevance of haplotypes composed of these loci and MDs. SPSS version 13.0 statistical software (SPSS, Inc., Chicago, IL, USA) was used for statistical data processing, with $\mathrm{P}<0.05$ considered to indicate a statistically significant difference.

Ethical and clinical test specification. The stages of the research program were evaluated by the Ethics Committee of Henan Provincial People's Hospital, and all participants in the study provided signed informed consent prior to the survey.

\section{Results}

Genotype frequencies and allele frequencies of the eight SNP loci near the $A B O$ gene. The tests revealed that the genotype distributions of the eight loci between the two groups met the Hardy-Weinberg genetic equilibrium law. Thus, the eight loci exhibited good population representation.

The results showed that among females of childbearing age in the Han population of Henan province, the frequency of the GG genotype and allele $\mathrm{G}$ in the rs657152 locus in the $\mathrm{ABO}$ blood group of the experimental group was higher than that in the control group. The frequency of the TT genotype of the experimental group was lower than that of the control group with a statistically significant difference $(\mathrm{P}<0.01)$. Relative risk analysis showed that the females who carried allele $\mathrm{G}$ exhibited an increased risk of MD [odds ratio $(\mathrm{OR})=0.602 ; 95 \%$ confidence interval $(\mathrm{CI}), 0.468-0.773]$. The difference in the distribution frequency of allele $\mathrm{T}$ of the rs495828 locus upstream of the ABO blood group gene between the experimental and control groups was statistically significant $(\mathrm{P}<0.05)$; females with allele $\mathrm{T}$ had a reduced risk of MDs $(\mathrm{OR}=1.372 ; 95 \% \mathrm{CI}, 1.026-1.835)$. The AA genotype frequency of the TRAF2 rs17250673 locus, downstream of the ABO blood group gene was higher in the experimental group than in the control group; the difference was statistically significant $(\mathrm{P}<0.05)$; pairwise comparison revealed that only 
Table I. Genotype and allele frequency distributions of eight SNP loci in the experimental and control groups.

\begin{tabular}{|c|c|c|c|c|c|c|c|c|c|c|}
\hline \multirow[b]{2}{*}{ SNP } & \multirow[b]{2}{*}{ Gene } & \multirow[b]{2}{*}{$\begin{array}{c}\text { Genotype/ } \\
\text { allele }\end{array}$} & \multicolumn{2}{|c|}{ Experimental group } & \multicolumn{2}{|c|}{ Control group } & \multirow[b]{2}{*}{$\chi^{2}$} & \multirow[b]{2}{*}{ P-value } & \multirow[b]{2}{*}{ OR } & \multirow[b]{2}{*}{$95 \% \mathrm{CI}$} \\
\hline & & & $\begin{array}{l}\text { Absolute } \\
\text { frequency }\end{array}$ & $\begin{array}{c}\text { Frequency } \\
(\%)\end{array}$ & $\begin{array}{l}\text { Absolute } \\
\text { frequency }\end{array}$ & $\begin{array}{c}\text { Frequency } \\
(\%)\end{array}$ & & & & \\
\hline \multirow[t]{5}{*}{ rs657152 } & $\mathrm{ABO}$ & $\mathrm{TT}$ & 46 & 0.184 & 58 & 0.232 & 26.979 & $<0.001^{\mathrm{a}}$ & & \\
\hline & & $\mathrm{TG}$ & 113 & 0.452 & 152 & 0.608 & & & & \\
\hline & & GG & 91 & 0.364 & 40 & 0.160 & 15.158 & $<0.001^{\mathrm{a}}$ & 0.349 & $0.204-0.596$ \\
\hline & & $\mathrm{G}$ & 295 & 0.590 & 232 & 0.464 & 15.922 & $<0.001^{\mathrm{a}}$ & 0.602 & $0.468-0.773$ \\
\hline & & $\mathrm{T}$ & 205 & 0.410 & 268 & 0.536 & & & & \\
\hline \multirow[t]{5}{*}{ rs495828 } & $\begin{array}{l}\text { Upstream } \\
\text { of ABO }\end{array}$ & GG & 152 & 0.608 & 130 & 0.520 & 4.942 & 0.089 & & \\
\hline & & GT & 89 & 0.356 & 104 & 0.416 & & & & \\
\hline & & TT & 9 & 0.036 & 16 & 0.064 & & & & \\
\hline & & $\mathrm{T}$ & 107 & 0.214 & 136 & 0.272 & 4.572 & $0.033^{\mathrm{a}}$ & 1.372 & $1.026-1.835$ \\
\hline & & G & 393 & 0.786 & 364 & 0.728 & & & & \\
\hline \multirow[t]{5}{*}{ rs7852970 } & TRAF2 & TT & 22 & 0.088 & 22 & 0.088 & 0.406 & 0.804 & & \\
\hline & & CT & 104 & 0.416 & 97 & 0.388 & & & & \\
\hline & & $\mathrm{CC}$ & 124 & 0.496 & 131 & 0.524 & & & & \\
\hline & & $\mathrm{C}$ & 352 & 0.704 & 359 & 0.718 & 0.238 & 0.625 & 1.071 & $0.814-1.407$ \\
\hline & & $\mathrm{T}$ & 148 & 0.296 & 141 & 0.282 & & & & \\
\hline \multirow[t]{5}{*}{ rs4448378 } & TRAF2 & GG & 5 & 0.020 & 5 & 0.020 & 0.041 & 0.979 & & \\
\hline & & $\mathrm{CG}$ & 67 & 0.268 & 65 & 0.260 & & & & \\
\hline & & $\mathrm{CC}$ & 178 & 0.712 & 180 & 0.720 & & & & \\
\hline & & $\mathrm{C}$ & 423 & 0.846 & 425 & 0.850 & 0.031 & 0.860 & 1.032 & $0.730-1.457$ \\
\hline & & $\mathrm{G}$ & 77 & 0.154 & 75 & 0.150 & & & & \\
\hline \multirow[t]{5}{*}{ rs10781522 } & TRAF2 & $\mathrm{AA}$ & 21 & 0.084 & 22 & 0.088 & 1.914 & 0.384 & & \\
\hline & & $\mathrm{AG}$ & 107 & 0.428 & 92 & 0.368 & & & & \\
\hline & & GG & 122 & 0.488 & 136 & 0.544 & & & & \\
\hline & & $\mathrm{G}$ & 315 & 0.702 & 364 & 0.728 & 2.789 & 0.095 & 1.266 & $0.960-1.670$ \\
\hline & & A & 149 & 0.298 & 136 & 0.272 & & & & \\
\hline \multirow[t]{5}{*}{ rs17250673 } & TRAF2 & $\mathrm{AA}$ & 10 & 0.040 & 5 & 0.020 & 7.312 & $0.026^{\mathrm{a}}$ & & \\
\hline & & $\mathrm{AG}$ & 50 & 0.200 & 74 & 0.294 & & & & \\
\hline & & GG & 190 & 0.760 & 171 & 0.684 & & & & \\
\hline & & G & 430 & $0 / .860$ & 416 & 0.832 & 1.504 & 0.220 & 0.806 & $0.571-1.138$ \\
\hline & & A & 70 & 0.140 & 84 & 0.168 & & & & \\
\hline \multirow[t]{5}{*}{ rs61754920 } & PNPLA7 & GG & 150 & 0.600 & 149 & 0.596 & 2.939 & 0.230 & & \\
\hline & & $\mathrm{AG}$ & 89 & 0.356 & 80 & 0.320 & & & & \\
\hline & & AA & 11 & 0.044 & 21 & 0.084 & & & & \\
\hline & & A & 111 & 0.222 & 122 & 0.244 & 0.677 & 0.411 & 1.131 & $0.843-1.517$ \\
\hline & & $\mathrm{G}$ & 389 & 0.778 & 378 & 0.756 & & & & \\
\hline \multirow[t]{5}{*}{ rs11137410 } & PNPLA7 & $\mathrm{CC}$ & 158 & 0.632 & 155 & 0.620 & 2.231 & 0.313 & & \\
\hline & & $\mathrm{CT}$ & 81 & 0.324 & 76 & 0.304 & & & & \\
\hline & & TT & 11 & 0.044 & 19 & 0.076 & & & & \\
\hline & & $\mathrm{T}$ & 103 & 0.206 & 114 & 0.228 & 0.712 & 0.399 & 1.138 & $0.842-1.538$ \\
\hline & & $\mathrm{C}$ & 397 & 0.794 & 386 & 0.772 & & & & \\
\hline
\end{tabular}

aP $<0.05$ was considered statistically significant. SNP, single nucleotide polymorphism; OR, odds ratio; CI, confidence interval; TRAF2, tumour necrosis factor cofactor 2; PNPLA7, patatin-like phospholipase domain containing 7.

the differences between the AG and GG genotypes were statistically significant $(\mathrm{P}<0.05)$. The differences of the intergroup allele distribution frequencies of rs17250673 were statistically insignificant (Table I). 
Table II. Genotype and allele frequency distributions of eight SNP loci in the experimental and control groups in the dominant and recessive models.

\begin{tabular}{|c|c|c|c|c|c|c|c|c|c|}
\hline \multirow[b]{2}{*}{ SNP } & \multirow[b]{2}{*}{ Gene } & \multicolumn{4}{|c|}{ Dominant model } & \multicolumn{4}{|c|}{ Recessive model } \\
\hline & & $\chi^{2}$ & P-value & OR & $95 \% \mathrm{CI}$ & $\chi^{2}$ & P-value & OR & $95 \% \mathrm{CI}$ \\
\hline rs657152 & $\mathrm{ABO}$ & 26.904 & $<0.001^{\mathrm{a}}$ & 3.005 & $1.964-4.596$ & 0.868 & 2.086 & 1.340 & $0.868-2.068$ \\
\hline rs495828 & ABO upstream & 2.063 & 0.151 & 1.831 & $0.793-4.255$ & 3.936 & $0.047^{\mathrm{a}}$ & 0.698 & $0.490-0.996$ \\
\hline rs7852970 & TRAF2 & 0.392 & 0.531 & 1.119 & $0.788-1.589$ & 0.000 & 1.000 & 1.000 & $0.539-1.857$ \\
\hline rs4448378 & TRAF2 & 0.039 & 0.843 & 1.040 & $0.705-1.534$ & 0.000 & 1.000 & 1.000 & $0.286-3.498$ \\
\hline rs10781522 & TRAF2 & 1.570 & 0.210 & 1.252 & $0.881-1.779$ & 0.025 & 0.873 & 1.052 & $0.563-1.967$ \\
\hline rs17250673 & TRAF2 & 3.597 & 0.058 & 0.684 & $0.461-1.014$ & 1.718 & 0.190 & 0.490 & $0.165-1.454$ \\
\hline rs61754920 & PNPLA7 & 3.339 & 0.068 & 1.992 & $0.940-4.225$ & 0.008 & 0.927 & 0.983 & $0.688-1.406$ \\
\hline rs11137410 & PNPLA7 & 2.270 & 0.132 & 1.787 & $0.832-3.838$ & 0.077 & 0.782 & 0.950 & $0.661-1.365$ \\
\hline
\end{tabular}

${ }^{a} \mathrm{P}<0.05$ was considered statistically significant. SNP, single nucleotide polymorphism; OR, odds ratio; CI, confidence interval; TRAF2, tumour necrosis factor cofactor 2; PNPLA7, patatin-like phospholipase domain containing 7.

Table III. Genotype and allele frequency distribution of the rs17250673 locus in the ultra-dominant model.

Ultra-dominant model

\begin{tabular}{lccccc}
\cline { 3 - 6 } SNP & Gene & $\chi^{2}$ & P-value & OR & $95 \%$ CI \\
\hline rs657152 & TRAF2 & 6.177 & $0.013^{\mathrm{a}}$ & 0.595 & $0.394-0.898$
\end{tabular}

${ }^{\mathrm{a}} \mathrm{P}<0.05$ was considered statistically significant. SNP, single nucleotide polymorphism; OR, odds ratio; CI, confidence interval; TRAF2, tumour necrosis factor cofactor 2 .

Allele frequencies of the eight SNP loci in the recessive and dominant models. To expand the sample size further and increase the differences among the genotypes, each locus was analysed under the dominant and recessive models. The results demonstrated that the difference in the genotype distribution frequency of the rs657152 locus of the $\mathrm{ABO}$ blood group gene between the two groups exhibited statistical significance in the dominant model; the TT or TG genotype-carrying population had a lower risk of MDs than the GG population (OR=3.005; 95\% CI, 1.964-4.596). The difference in the rs495828 locus genotype distribution frequency was statistically significant in the recessive model, with the TT or TG genotype-carrying population having higher risk of MDs than the GG population (OR=0.698; 95\% CI, 0.490-0.996). The other loci exhibited no statistically significant differences (Table II).

The three genotypes of the TRAF2 gene rs17250673 locus between the two groups had statistically significant differences in distribution frequency, whereas the dominant and recessive models had statistically insignificant differences. However, given the difference in AG and GG genotype frequencies, the difference was analysed in the ultra-dominant model and found to be significant (Table III), indicating that the pathogenic effect of the heterozygous genotype AG at this locus was greater than that of any homogeneous genotype. This result may be attributed to the increased interaction between genes due to the heterozygous state and that heterozygosity itself is the fundamental reason for hybrid advantages.

Haplotype analysis. SHEsis software was used to analyse the linkage disequilibrium and haplotype frequency of all loci. Out of the eight loci, only S3 and S52 loci were in a region of linkage disequilibrium $\left(\mathrm{D}^{\prime}=0.090\right)$ and could be used to build haplotypes AA and GG. The P-values of the two loci in the experimental and control groups were both 0.539 , thus indicating statistical insignificance.

\section{Discussion}

MDs are a common gynaecological diseases that not only affect the health and quality of life of females but also increase the potential risks of certain gynaecological diseases. For example, menarche at an earlier age increases the risk of breast cancer (1) and endometrial cancer (2), and late menarche increases the risk of Alzheimer's disease (3) and osteoporosis (4). The occurrence of MDs is affected by various factors. Taylor et al (5) studied the progesterone receptor gene and 37 SNP loci in upstream and downstream regions and found that the polymorphisms of the progesterone receptor gene were associated with a young menarche age and excessively short menstrual cycle. Furthermore, the risk for PMS has been found to be higher in identical twins than in fraternal twins (6), and mutation of the FSH-R gene may lead to amenorrhoea, infertility or premature ovarian failure (7). These results indicate that genetic susceptibility is significant to MD occurrence.

Previous reports have described the associations of ABO blood group with ovarian reserve functions and infertility (10-12). In 2010, experts from Yale University and Albert Einstein Medical College proposed at the 66th ASRM Annual Meeting (13) that type O blood was associated with infertility. Binder et al $(14,15)$ found that pregnant females with type A blood were more prone to suffer from symptoms of ovarian hyperstimulation than females with type O blood. Nejat et al (10) found that antigen A may be a protective factor 
of ovarian reserve capacity and that type O blood decreased the risk of ovarian reserve capacity more than blood of types A and AB. Chen et al (9) performed a genome-wide association study, identified a predisposing gene for the polycystic ovary syndrome located in 9q33.3 and found that polycystic ovary syndrome was normally accompanied by MDs. The ABO gene is located on chromosome $9 \mathrm{q} 34$.2; thus, the association of the blood group system with MDs may be related to the ABO gene. Specific genes that are associated with MDs may be linked with the ABO gene and located near the chromosome $9 q 34.2$. An unknown mutation close to the ABO gene might be associated with MDs, thus explaining the correlation of the ABO blood group system with the abnormalities of menstrual and reproductive systems from a theoretical basis. Thus, the ABO blood group gene and eight loci located in upstream and downstream related genes were examined. Whether these SNPs are correlated with MDs was evaluated in this study.

The ABO blood group gene is located on chromosome 9q34.1-q34.2 (19), which contains seven exons with lengths of $\sim 18 \mathrm{~kb}$ and six introns with lengths of $\sim 19,514 \mathrm{bp}$. The product of the ABO gene is glycosyltransferase, which controls the biosynthesis of ABO blood group antigens. The chemical structure of the ABO antigens comprises glycoproteins. The product of the A gene is N-acetyl galactosyltransferase, which links $\alpha-\mathrm{N}$-acetylgalactosamine with antigen $\mathrm{H}$, an $\mathrm{ABO}$ antigen precursor, thus producing the $\mathrm{A}$ antigenic determinant. The product of the $\mathrm{B}$ gene is galactosyltransferase, which links acetyl- $\alpha$-D-galactose with antigen $\mathrm{H}$, thus generating the $\mathrm{B}$ antigenic determinant. In type O blood, several inactive genes that potentially encode non-active transferase were found. Type $\mathrm{O}$ blood is the unaltered precursor of $\mathrm{A}$ or $\mathrm{B}$, and the $\mathrm{OO}$ gene does not generate a specific antigen (20).

It has been assumed that a definite link exists between the ABO blood group and disease. Henry and Samuelsson (21) made the following summary of the correlation of $\mathrm{ABO}$ polymorphisms and diseases: "the bacterial infection tended to infringe the A type, the viral infection was usually associated with $\mathrm{O}$ type, the tumour was most associated with A type, and the autoimmunity was associated with type O blood'. In the present study, the correlation analysis of the ABO-gene chromosomal-regional allele distribution frequencies and genotypes showed that the three genotypes of the rs657152 locus of the ABO gene exhibited statistically significant differences between the experimental and control groups. The frequency of the GG genotype was higher in the experimental group than in the control group, and people carrying allele $G$ suffered from an increased risk of MD. A previous study reported the association of this locus with a variety of diseases, and an increased risk of pancreatic cancer and gastric ulcers in populations with allele $\mathrm{G}$ at this locus (22). The levels of inflammatory cytokines, intercellular adhesion molecules and E-selectin are increased in patients with pancreatic cancer carried on the $\mathrm{G}$ allele, indicating that allele $\mathrm{G}$ is the marker gene in Caucasians with type $\mathrm{O}$ blood; the $\mathrm{G}$ allele at this locus is a risk allele (22). Several key proteins associated with follicle development and menstruation, such as the FSH-R and luteinizing hormone receptor, are glycosyltransferase proteins. This explains the association of the ABO blood group with female reproductive endocrinology and the association between the $\mathrm{O}$ blood type and infertility. Glycosyltransferase blood type antigens, which exist in females without type $\mathrm{O}$ blood, might have an important role in the development of ovarian endocrine cells. Williams and Stanley (23) found that mice with lower ovulation rates lacked a certain oocyte-specific glycosyltransferase.

The rs495828 locus, which is located upstream of the ABO gene, exhibited statistical insignificance among the three genotypes but exhibited a statistical significance between alleles $\mathrm{T}$ and $\mathrm{G}$. Carriers of $\mathrm{T}$ alleles exhibited a reduced risk of MD occurrence. It has been reported that this locus is associated with the activity of angiotensin-converting enzyme (ACE) (24). ACE is the key enzyme of the renin-angiotensin system, which converts angiotensin I into angiotensin II; the latter could lead to follicular atresia at various stages, thus resulting in anovulation. This locus may cause MDs by influencing ACE activities.

Tumour necrosis factor (TNF)- $\alpha$ is a pleiotropic cytokine that is involved in cell proliferation and apoptosis and plays an important regulatory role during follicle development, ovulation, corpus luteum formation and steroid hormone generation. The TRAF family is a class of plasmalemma adapter proteins that directly binds to the intracellular region of cell surface receptors. TRAFs interact with the members of the TNF receptor superfamily, thus causing a series of downstream signalling cascades, activating downstream signalling molecules, such as I $\mathrm{KB}$ kinase and mitogen-activated protein kinase, and ultimately affecting the activities of transcription factors NF- $\kappa \mathrm{B}$ and AP-1 (25). In TNFR and IL-IR/Toll receptor-mediated signalling pathways, TRAF2, TRAF5 and TRAF6 play key roles in the activation of the IкB kinase complex and JNK (26).

The rs17250673 locus of the TRAF2 gene exhibits statistical significance in the genotype frequency distributions and statistically significant differences in the ultra-dominant model. TRAF2 locus mutation may affect the expression level or functions of TRAF2, thus influencing the biological effects of various factors during the expression of TRAF2 regulatory genes and TNF signalling pathways, causing anabolism disorder of ovarian steroid hormones and affecting follicular development and ovulation. This indicates that females with the heterozygous genotype of TRAF2 rs17250673 suffer from increased MD susceptibility.

In summary, the ABO blood group gene and TRAF2 gene may be predisposing genes of MDs since the chromosome region at which the $\mathrm{ABO}$ blood group gene is located is associated with genetic causes of MDs. However, a large-sample, multi-regional study of other related loci in this region is required to elucidate the pathogenesis of MDs and obtain results with theoretical significance and application values.

\section{Acknowledgements}

This study was supported by the National Natural Science foundation of China (no. 30770828).

\section{References}

1. Tao P, Hu YY, Huang Y and Li JY: Risk factors of breast cancer in Asian women: a meta-analysis. Zhonghua Liu Xing Bing Xue Za Zhi 32: 164-169, 2011 (In Chinese). 
2. Reis $\mathrm{N}$ and Beji NK: Risk factors for endometrial cancer in Turkish women: results from a hospital-based case-control study. Eur J Oncol Nurs 13: 122-127, 2009.

3. Liu YZ, Li J, Pan R, et al: Genome-wide copy number variation association analyses for age at menarche. J Clin Endocrino Metab 97: 2133-2139, 2012

4. Francucci CM, Romagni P, Camilletti A, et al: Effect of natura early menopause on bone mineral density. Maturitas 59: 323-328, 2008.

5. Taylor KC, Small CM, Epstein MP, et al: Associations of progesterone receptor polymorphisms with age at menarche and menstrual cycle length. Horm Res Paediatr 74: 421-427, 2010.

6. Jahanfar S, Lye MS and Krishnarajah IS: The heritability of premenstrual syndrome. Twin Res Hum Genet 14: 433-436, 2011.

7. Lussiana C, Guani B, Mari C, Restagno G, Massobrio M and Revelli A: Mutations and polymorphisms of the FSH receptor (FSHR) gene: clinical implications in female fecundity and molecular biology of FSHR protein and gene. Obstet Gynecol Surv 63: 785-795, 2008

8. Dewailly D, Gronier H, Poncelet E, et al: Diagnosis of polycystic ovary syndrome (PCOS): revisiting the threshold values of follicle count on ultrasound and of the serum AMH level for the definition of polycystic ovaries. Hum Reprod 26: 3123-3129, 2011.

9. Chen ZJ, Zhao H, He L, et al: Genome-wide association study identifies susceptibility loci for polycystic ovary syndrome on chromosome 2p16.3, 2p21 and 9q33.3. Nat Genet 43: 55-60, 2011

10. Nejat EJ, Jindal S, Berger D, Buyuk E, Lalioti M and Pal L: Implications of blood type for ovarian reserve. Hum Reprod 26: 2513-2517, 2011.

11. Nejat EJ, Jindal S, Berger D, et al: Implications of blood type for ovarian reserve. Human Reproduction 26: 2513-2517, 2011.

12. Timberlake KS, Foley KL, Hurst BS, Matthews ML, Usadi RS and Marshburn PB: Association of blood type and patient characteristics with ovarian reserve. Fertil Steril 100: 1735-1739, 2013.

13. WebMD Health News: Blood type O linked to fertility problems study: Women with type O blood more likely to have lower egg reserves. http://www2.your4state. com/webmd/infertility/story/Blood-Type-O-Linked-to-FertilityProblems/uQq2LT7o40yhwF6-va0aAw.cspx. Accessed January $14,2015$.
14. Binder H, Flegel WA, Emran J, et al: Blood group A: an overseen risk factor for early-onset ovarian hyperstimulation syndrome? Reprod Biomed Online 17: 185-189, 2008.

15. Binder H, Flegel WA, Emran J, et al: Association of blood group A with early-onset ovarian hyperstimulation syndrome. Transfus Clin Biol 15: 395-401, 2008.

16. Montoya JS, Cabezza AH, Rojas OM, Navarrete RC and Keever MAV: Menstrual disorders in adolescents. Bol Med Hosp Infant Mex 69: 60-72, 2012.

17. International HapMap Project. http://hapmap.ncbi.nlm.nih.gov/. Accessed October 7, 2012.

18. SHEsis software. http://analysis.bio-x.cn/myAnalysis.php. Accessed October 7, 2012.

19. Pelzer U, Klein F, Bahra M, et al: Blood group determinates incidence for pancreatic cancer in Germany. Front Physiol 24: 118, 2013.

20. Daniels G and Reid ME: Blood groups: the past 50 years. Transfusion 50: 281-289, 2010 .

21. Henry S and Samuelsson B: ABO polymorphisms and their putative biological relationships with disease. In: Human Blood Cells: Consequences of Genetic Polymorphisms and Variations. King M-J (ed). Imperial College Press, London. pp15-pp103, 2000.

22. Amundadottir L, Kraft P, Stolzenberg-Solomon RZ, et al. Genome-wide association study identifies variants in the ABO locus associated with susceptibility to pancreatic cancer. Nat Genet 41: 986-990, 2009.

23. Williams SA and Stanley P: Oocyte-specific deletion of complex and hybrid $\mathrm{N}$-glycans leads to defects in preovulatory follicle and cumulus mass development. Reproduction 137: 321-331, 2009.

24. Gassó P, Mas S, Álvarez S, et al: A common variant of the ABO gene protects against hypertension in a Spanish population. Hypertens Res 35: 592-596, 2012.

25. Zheng C, Kabaleeswaran V, Wang Y, Cheng G and Wu H: Crystal structures of the TRAF2: cIAP2 and the TRAF1: TRAF2: cIAP2 complexes: affinity, specificity, and regulation. Mol Cell 38: 101-113, 2010.

26. Melas IN, Mitsos A, Messinis DE, Weiss TS, Rodriguez JS and Alexopoulos LG: Construction of large signaling pathways using an adaptive perturbation approach with phosphoproteomic data. Mol Biosyst 8: 1571-1584, 2012. 